\title{
Injury hospitalizations among American Indian youth in Washington
}

\author{
Shannon J Johnson, Marianne Sullivan, David C Grossman
}

\begin{abstract}
Objective-To determine the rate and causes of hospitalizations for injury among American Indian and Alaska Native (AI/AN) youth in the state of Washington, and to compare this with the rate of hospitalizations for injury among youth of all races.
\end{abstract}

Methods-Subjects were aged 0-19 years and were admitted to civilian hospitals for care of an injury (International Classification of Diseases N codes 800995) in Washington between 1990 and 1994. Deaths occurring in the prehospital setting and emergency department are not included. Using several fields of identifying information, the Washington state hospital discharge database was linked with the Indian Health Service (IHS) patient registration database to identify AI/AN youth. Denominator data included the total age specific IHS user population for American Indians and US Census derived population estimates. Incidence ratios (IRs) were calculated to compare rates of hospitalization between AI/AN youth and all youth in Washington.

Results-A total of 694 and 29048 hospitalizations for injury were identified for AI/AN youth and all races, respectively. The rate of hospitalization for injuries among AI/AN youth was 507 discharges per 100000 youth $($ IR $=1.30 ; 95 \%$ confidence interval (CI) 1.20 to 1.40 . The leading mechanism of injury was motor vehicles (IR 1.73, CI 1.49 to 2.01), followed by falls (IR 0.95 , CI 0.79 to 1.15 ), and poisoning (IR 1.20, CI 0.80 to 1.78). The disparity was greater for intentional injuries (IR 1.71, CI 1.44 to 2.04). The highest IR for all unintentional injuries was for injuries from fire (IR 2.35, CI 1.42 to 3.87). AI/AN children aged 15-19 had the greatest disparity for rates of injury hospitalization (IR 1.4, CI 1.25 to 1.56).

Conclusion-AI/AN youth in Washington had a higher hospitalization rate for injury compared with all youth in the state. Disparities were greatest for injuries related to motor vehicles and assaults. When linked, hospital discharge data can be used for surveillance of AI/AN hospitalizations.

(Injury Prevention 1999;5:119-123)

Keywords: Indians, North American; suicide, attempted; epidemiology
Injuries are a leading cause of childhood morbidity and mortality throughout the world. Although there has been substantial declines in the rates of injury deaths among children in developed nations, injuries remain the principal threat to the health and welfare of children and adolescents. ${ }^{1}$

Rates of injuries are known to be higher among indigenous peoples in North America, Australia, and New Zealand..$^{2-7}$ In the United States, American Indians and Alaska Natives (AIs/ANs) also suffer from injury morbidity and mortality at higher rates than other races. ${ }^{8}$ Hospitalization rates for injury among AIs/ANs have been published for the Indian Health Service (IHS) service population, but these analyses have not specifically focused on children. ${ }^{9}$ Furthermore, these analyses have used IHS hospital discharge data and may miss $\mathrm{AI} / \mathrm{AN}$ patients who receive hospital care outside of the IHS system, especially if the payer is other than the IHS. State hospital discharge registries represent another potential source of data for surveillance of hospitalizations, but many do not contain racial or ethnic identification data. If they do, the potential also exists for racial misclassification. ${ }^{10}$

The severity and cost of injury hospitalizations to AIs/ANs have not been well described. In many areas, where IHS hospitals care for most hospitalized patients, there is no billing for registered $\mathrm{AIs} / \mathrm{ANs}$ and therefore no charges or costs are calculated. Only those patients receiving care in contract health facilities outside of the IHS system (often the most severely injured) receive a bill. As Indian tribes and Native corporations assume more responsibility for their health care under self determination laws, leading to fixed government contracts, most hospitalization costs will be borne directly by tribes. ${ }^{11}$ As a result, tribes will have an increasing financial incentive to reduce preventable injuries and illnesses among their membership. Hence, local surveillance mechanisms using available data will be essential.

The purpose of this study was to determine the incidence of hospitalizations for injuries among IHS eligible AI/AN youth in the state of Washington for the years 1990 to 1994 . By linking state hospital discharge and IHS patient registration data, we sought to compare injury hospitalization rates to those of the state's entire youth population. Finally, we sought to determine the severity and total costs of these injuries.

\section{Methods}

SOURCES OF DATA

The Comprehensive Hospital Abstract Reporting System (CHARS), the state hospital 
discharge registry, contains a record for each individual discharged from a civilian hospital in Washington state. Included in the database is information on patient characteristics such as age, sex, type of injury, mechanism of injury, length of hospital stay, intent of injury, cost of hospitalization, and payer. However, CHARS does not contain ethnic or racial identifying information.

The IHS is charged with providing direct and contracted health care services to enrolled members of federally recognized tribes, and maintains a database of members who are registered for IHS services. There are no inpatient IHS facilities in the state of Washington; all inpatient care is provided in private hospitals under contract with the IHS or the tribe. Since the CHARS database does not contain information on race or ethnicity, $\mathrm{AI} / \mathrm{AN}$ race was assessed using the IHS patient registry. The registry includes the patient's full name (including alias names used by the patient), address, date of birth, gender. The IHS patient registration file was linked to the CHARS database to determine which individuals hospitalized for injury were of AI or AN origin.

We linked the Washington state hospital discharge database with the IHS database by computer, using the first two initials of the patient's first name and last name, gender, and date of birth using a computerized matching program created for this purpose. Alias names, when present, were also used for possible matching. Only exact matches on all of these fields were allowed.

STUDY POPULATION

The numerator consisted of all IHS enrolled children and adolescents (aged 0-19 years) who linked to the CHARS database for the years 1990 to 1994 , with a principal diagnosis of injury (International Classification of Diseases, ninth revision (ICD-9)-CM N codes 800-995) averaged annually over the five year period. The denominator for American Indian children included all IHS enrolled members aged 0-19 reported to be living in the state of Washington at the study midpoint, in June 1992.

Rates of injury hospitalizations among $\mathrm{AI} / \mathrm{AN}$ youth were compared with those of all youth in the state using the statewide hospital discharge database. The denominator used for all youth in the state was the State of Washington Office of Financial Management's inter-

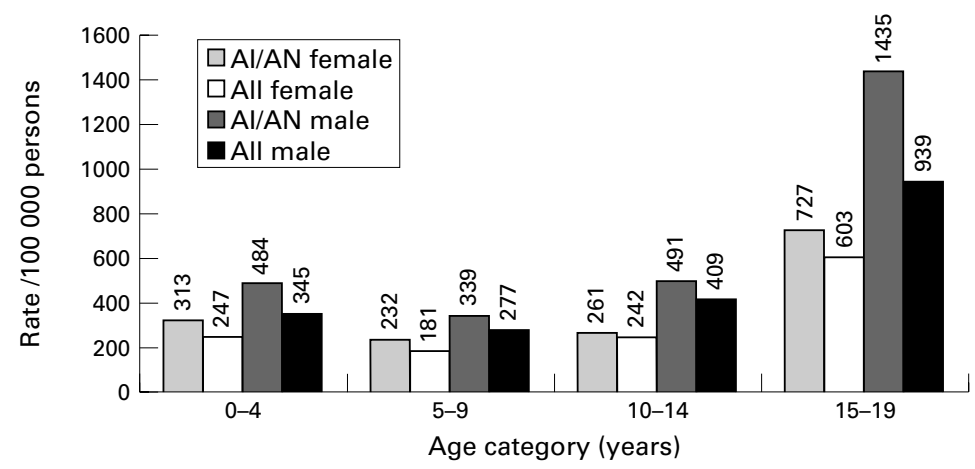

Figure 1 Youth injury hospitalizations rates by age, sex, and race. censal population estimate of $0-19$ year olds residing in Washington in 1992; the midpoint of the study period. The injury severity score, ${ }^{12}$ a widely recognized tool to characterize anatomic injury severity, was derived for each subject using ICDMAP, a computerized program that converts ICD-9-CM coded diagnoses into abbreviated injury scale and composite injury severity scores. The abbreviated injury scale quantifies the extent of injury to each of six body regions. The injury severity score is computed by summing the squares of the three highest abbreviated injury scale scores in the three body regions with the highest abbreviated injury scale scores. An injury severity score greater than 15 is considered to represent a severe injury and scores of 9-15 are of moderate severity. ${ }^{12}$

External cause of injury codes (ICD-9-CM E codes), included in the hospital discharge database, were categorized as proposed by the Centers for Disease Control, with modifications to stratify more of the mechanisms grouped as "other". ${ }^{13}$ We excluded the following $\mathrm{E}$ codes related to adverse effects of care: E870-876 , E878-879 , E-930-949.9, E929, E959, E969, E977, E989, E999. Mechanism of injury (E codes) were available for $659(92.2 \%)$ AI/AN hospitalizations and for 27668 (95.2\%) all race hospitalizations.

Charges in the discharge database were used as a proxy for cost and reflected charges only for hospital care; pre-hospital charges and professional fees were excluded.

\section{Results}

INJURY DISCHARGE RATES

There were a total of 694 and 29048 injury hospitalizations among AI/AN youth and all youth residing in Washington, respectively, between 1990 and 1994. The population of IHS enrolled 0-19 year old AIs/ANs was estimated at 26 659. According to census data, $34736 \mathrm{AI} / \mathrm{AN}$ youth and $14858630-19$ year old youth of all races resided in Washington in 1992.

While IHS enrolled youth make up $1.8 \%$ of the Washington's youth population, they represented $2.4 \%$ of injury hospitalizations. AI/AN youth had an annualized injury hospitalization rate of 507 per 100000 population compared to 391 per 100000 for all youth statewide (incidence ratio (IR) 1.30, 95\% confidence interval (CI) 1.20 to 1.40 ). The disparity between groups was considerably lower for unintentional injuries (IR 1.19, CI 1.09 to 1.30) than for intentional injuries (IR 1.71, CI 1.44 to 2.44 ).

The highest rates of injury hospitalization occurred among AI/AN males aged 15-19 years (1435/100 000 population). Although 15-19 year old AI/AN males compose $22 \%$ of the AI/AN youth population, $45 \%$ of $\mathrm{AI} / \mathrm{AN}$ hospitalizations occurred in this group. When stratified by age and gender, AIs/ANs appear to have consistently higher rates of injury hospitalization in every group (fig 1 ).

Although AI/AN unintentional injury hospitalizations were most frequently for motor 
Table 1 Unintentional injury hospitalization rates in AI/AN youth by mechanism, Washington State 1990-94

\begin{tabular}{lrrll}
\hline Mechanisms of injury & AI/AN & All races & Incidence ratio & $\begin{array}{l}\text { 95\% Confidence } \\
\text { interval }\end{array}$ \\
\hline Motor vehicles & & & & \\
$\quad$ Traffic related & 127.8 & 73.9 & 1.73 & 1.49 to 2.01 \\
$\quad$ Pedal cyclist & 16.1 & 14.2 & 1.13 & 0.74 to 1.72 \\
$\quad$ MV, non-traffic & 9.5 & 8.7 & 1.10 & 0.63 to 1.90 \\
Falls & 79.6 & 83.5 & 0.95 & 0.79 to 1.15 \\
Struck by/against & 27.8 & 26.5 & 1.05 & 0.76 to 1.45 \\
Poisoning & 18.3 & 15.3 & 1.20 & 0.80 to 1.78 \\
Cut/pierce & 13.9 & 11.2 & 1.24 & 0.78 to 1.95 \\
Fire/flames & 11.7 & 5.0 & 2.35 & 1.42 to 3.87 \\
Hot object/scald & 10.2 & 10.7 & 0.96 & 0.56 to 1.62 \\
Other transport & 9.5 & 6.3 & 1.50 & 0.87 to 2.60 \\
Environmental factors & 6.6 & 7.9 & 0.83 & 0.43 to 1.60 \\
Firearm & 5.1 & 3.5 & 1.46 & 0.69 to 3.10 \\
Suffocation & 5.1 & 5.3 & 0.96 & 0.46 to 2.03 \\
Machine & 3.6 & 3.0 & 1.23 & 0.51 to 2.99 \\
Unintentional total & 373.2 & 313.8 & 1.19 & 1.09 to 1.30 \\
\hline
\end{tabular}

Rates expressed as hospital discharges per 100000 youth

$\mathrm{MV}=$ motor vehicle .

vehicle injuries (26\% of admissions), falls $(17 \%)$, and poisonings $(12 \%)$, falls were the leading mechanism (22\%) among all Washington youth, followed by motor vehicles $(19 \%)$ and poisoning (12\%) (table 1). AI/AN males were over 1.5 times more likely to be hospitalized for motor vehicle occupant injuries than females, and over four times as likely to be hospitalized for motorcycle injuries. Among unintentional injuries, the greatest IR was for injuries related to fire and flames (IR 2.35, CI 1.42 to 3.87 ).

Rates of intentional injuries and assaults were higher among AIs/ANs compared with all youth (IR $1.71,95 \%$ CI 1.44 to 2.04 ) (table 2). The greatest disparity in rates for AIs/ANs, compared with the state as a whole, was for assaultive injuries. Incidence ratios for self inflicted injuries were about half of those for assaults. $\mathrm{AI} / \mathrm{AN}$ males were nearly twice as likely to be hospitalized for intentional injuries than $\mathrm{AI} / \mathrm{AN}$ females, and nearly five times as likely to be hospitalized for assault related injuries. However, AI/AN females were more than twice as likely to be hospitalized for self inflicted injuries than their male counterparts. The leading mechanism of self inflicted injury was poisoning $(75 \%)$, followed by cutting or piercing $(10 \%)$. The most frequently reported mechanisms in assault hospitalizations were being struck (28.2\%), child battering/ maltreatment $(25.4 \%)$, and firearms $(19.7 \%)$.

During the five year study period, 10 injury hospitalizations resulted in death among $\mathrm{AI} / \mathrm{AN}$ youth after admission, eight of which

Table 2 Intentional injury hospitalization rates in AI/AN youth by mechanism, Washington State 1990-94

\begin{tabular}{lrrll}
\hline Mechanisms of injury & AI/AN & All races & Incidence ratio & $\begin{array}{l}\text { 95\% Confidence } \\
\text { interval }\end{array}$ \\
\hline Self inflicted injuries & & & & \\
$\quad$ All mechanisms & 42.4 & 35.5 & 1.19 & 0.92 to 1.55 \\
$\quad$ Cut/pierce & 4.4 & 2.8 & 1.58 & 0.70 to 3.56 \\
$\quad \begin{array}{l}\text { Poisoning } \\
\text { Assaults }\end{array}$ & 32.1 & 30.5 & 1.05 & 0.78 to 1.42 \\
$\quad$ All mechanisms & 51.1 & 19.1 & 2.67 & 2.10 to 3.40 \\
$\quad$ Struck by/against & 14.6 & 6.6 & 2.22 & 1.42 to 3.47 \\
$\quad$ Child maltreatment & 13.1 & 4.0 & 3.37 & 2.09 to 5.42 \\
$\quad$ Firearm & 10.2 & 3.9 & 2.58 & 1.51 to 4.42 \\
$\quad$ Cut/pierce & 5.8 & 2.9 & 2.03 & 1.00 to 4.11 \\
Total intentional & 93.5 & 54.6 & 1.71 & 1.44 to 2.04 \\
\hline
\end{tabular}

Rates expressed as hospital discharges per 100000 population. were related to motor vehicles. Poisoning was the mechanism for one in-hospital death, and one death was unspecified. Seven of the deaths were in the 15-19 age category.

Despite higher rates of injury hospitalizations among AI/AN youth, we found no significant differences between the mean injury severity score or length of stay between the two groups (AI/AN $8.4 v$ state 8.5 ). AI/AN males had a significantly higher mean injury severity score (male $9.2 v$ female 6.9, $\mathrm{p}=0.018$ ) compared with females. There were no other significant differences within the AI/AN group or between groups.

Hospital charges during the five year study period for the $694 \mathrm{AI} / \mathrm{AN}$ injury hospitalizations totaled $\$ 5113733$. The mean and median charges per injury hospitalization were $\$ 7356$ and $\$ 4142.93$ respectively and did not differ substantially between the two groups.

Motor vehicle injuries led to the highest cumulative charges (\$1560 739.80) with a mean charge per case $\$ 9180$. Injury by fire and flames had the second highest five year total charge (\$643 353) but the highest mean charge at $\$ 37844$. Charges for fall related injuries were one half million dollars, with a mean of $\$ 4526$.

Medicaid was the leading primary payer of hospital charges, accounting for half of the AI/AN hospitalizations, followed by private insurance $(28 \%)$, and the IHS $(11.2 \%)$. Among all youth, private insurance was the primary payer for $31 \%$ of the hospitalizations, followed by Medicaid (22\%), and health care service contractors $(19.5 \%)$.

\section{Discussion}

Our study found that AI/AN youth in the state of Washington have a significantly higher rate of injury related hospitalization when compared with all youth in the state of Washington. This disparity appears to related to a few key mechanisms of injury - intentional, fire related, and motor vehicle occupant injuries.

Motor vehicle injuries were the most prominent cause of injury hospitalizations among $\mathrm{AI} / \mathrm{AN}$ youth, but ranked second for all youth in the state. Other studies using hospital discharge data have also found falls to be the leading cause for all youth, with motor vehicles the leading mechanism only in the 15-19 year age group. ${ }^{14-16}$ One other population based study of childhood injuries in an American Indian community also demonstrated that motor vehicles are the leading cause of hospitalization for AI/AN youth, followed by falls. ${ }^{17}$ While few studies have compared injury discharge rates among racial and ethnic groups, ${ }^{18} 19$ we are unaware of any other large, detailed population based studies that have compared injury related hospital discharge rates between $\mathrm{AI} / \mathrm{AN}$ youth and other ethnic groups.

Variations in rates of injury among population groups are likely related to disproportionate differences in known risk factors. For example, a study on residential house fires in one Native community, reports a high preva- 
lence of risk factors such as smoking combined with alcohol, smoking in bed, and use of wood stoves. ${ }^{20} \mathrm{~A}$ study of risk factors for motor vehicle injury using statewide traffic accident database linked to the IHS database, concluded that $\mathrm{AI} / \mathrm{AN}$ drivers involved in rural crashes were much less likely to be wearing seat belts, and more likely to be alcohol impaired at the time of a crash compared to non-Indian drivers involved in crashes in rural areas. ${ }^{21}$ Other studies have also confirmed the prominent role of alcohol in motor vehicle injuries among American Indians. ${ }^{22} 23$

The relatively limited role of the IHS as the primary payer for the AI/AN hospitalizations reflects several factors. First, the IHS and tribes will usually seek other third party financing for medical care before using its own contract funds for hospital care. Since the IHS considers itself as the payer of last resort, private or Medicaid is first used, if possible. In many other areas of the United States, where the IHS is providing direct inpatient care, much of the cost is borne by the IHS.

\section{LIMITATIONS}

Hospital discharge databases have some limitations for injury surveillance. They have a relatively limited scope of clinical detail, may have inconsistent $\mathrm{E}$ coding, or double counting of patients transferred or readmitted for the same injury. ${ }^{24}$ In addition, this type of database only provides information on injuries requiring hospitalization, but excludes injury deaths occurring at the scene or in the emergency department.

Another potential limitation of this study is that the use of IHS eligibility as a measure of $\mathrm{AI} / \mathrm{AN}$ ethnicity is likely to underestimate the total population of AI/AN youths who are hospitalized. Many who identify themselves as $\mathrm{AI} / \mathrm{AN}$ are not enrolled in IHS because they never enrolled in IHS or because they are ineligible for services. American Indians living in urban areas are less likely to be enrolled in IHS because benefits may not be portable if they are not living on or near their tribal home, resulting in a disproportionate number of rural Indians in our study population.

Although these general patterns of injury may be consistent with other studies, we recognize that significant variations in disease and injury epidemiology exist among IHS service areas and that our findings may not be generalizable to other regions of North America or to other international indigenous groups. ${ }^{25}$

We believe that a strength of this study is the novel linkage of IHS enrollment files to state hospital discharge databases to ascertain the epidemiology of hospitalizations. This can prove to be an important tool for the future as analysts attempt to better define epidemiology of illness and injury among AI/AN youth.

PREVENTION PROGRAMS

Beginning in 1982, the IHS injury prevention program has assisted Indian communities with the development of injury prevention strategies. ${ }^{26}$ In 1990 Congress began appropriating funds to the 12 IHS areas specifically for injury prevention programs and training. Despite the huge burden of injury on the health of AIs/ANs, injury prevention constitutes a very small proportion of funds of the IHS budget.

The passage of the Indian SelfDetermination and Education Assistance Act (PL 93-638) in 1976 has led to the current trend for tribes taking responsibility for operating their own health care organizations and the devolution of the IHS. ${ }^{27}$ Tribal leaders will need to be informed of the injury epidemiology in their community, as well as effective available strategies for primary prevention and control. The unique information obtained from the linkage of databases, as demonstrated in this study, can ultimately be useful toward these ends.

We express our sincere appreciation to Robert Soderberg, Doni Wilder, Dee Robertson, and the delegates of the Northwest Portland Area Indian Health Board for their assistance and advice regarding this project.

This project was funded, in part, by a grant from the Centers for Disease Control and Prevention (R49/CCR002570).

1 Rivara FP, Grossman DC. Prevention of traumatic deaths to children in the United States: how far have we come and where do we need to go? Pediatrics 1996;97:791-7.

2 Silva DT, Ruben AR, Wronski I, et al. Excessive rates of childhood mortality in the Northern Territory, 1985-94. F Paediatr Child Health 1998;34:63-8.

3 MacKellar A. Deaths from injury in childhood in Western Australia 1983-1992. Med $\mathcal{F}$ Aust 1995;162:238-42.

4 Irving LM, Marshall SW, Norton RN. Motor vehicle occuIrving LM, Marshall SW, Norton RN. Motor vehicle occu-
pant injuries in New Zealand children, 1981-90. N Z Med pant injuries in New

5 Roberts I, Norton R, Hassall I. Child pedestrian injury 1978-87. N Z Med f 1992;105:51-2.

6 MacWilliam L, Mao Y, Nicholls E, et al. Fatal accidental childhood injuries in Canada. Can f Public Health 1987;78: 129-35.

7 Moffatt ME. Unmet needs of children in Canada's north: nutrition, injury prevention and problems of adolescents Arctic Med Res 1991;suppl:554-7.

8 Baker SP, O'Neill B, Ginsburg MJ, et al. The injury fact book. 2nd Ed. New York: Oxford University Press, 1992

9 Indian Health Service. Injuries among American Indians and Alaska Natives 1990. Rockville, MD: IHS, 1990.

10 Sugarman JR, Soderberg R, Gorden JE, et al. Effects of racial misclassification of American Indians on injury rates in Oregon, 1989-1990. Am f Public Health 1993;83:681-4.

11 Amendments to the Indian Health Care Improvement Act of 1992. Public Law No 102-537.

12 Mackenzie EH, Steinwachs DM, Shanker BS. Classifying trauma severity based on hospital discharge diagnosis. Validation of an ICD-N-CM to AIS-85 conversion table. Med Care 1989;4:412-22.

13 Centers for Disease Control and Prevention. Recommended framework for presenting injury mortality data. Morb Mortal Wkly Rep 1997;46:7.

4 Gallagher SS, Finison K, Guyer B, et al. The incidence of injuries among 87000 Massachusetts children and adolescents: results of the 1980-81 statewide childhood injury prevention program surveillance system. Am f Public Health 1984;74:1340-7.

15 Runyan CW, Kotch JB, Margolis LH, et al. Childhood injuries in North Carolina: a statewide analysis of hospitalizations and deaths. Am f Public Health 1985;75:1429-32.

16 Marganitt B, Mackenzie EJ, Deshpande JK, et al. Hospitalizations of traumatic injuries among children in Maryland: trends for incidence and severity: 1979-1988. Pediatrics 1992;89:608-13.

17 Berger LR, Kitzes J. Injuries to children in a Native American community. Pediatrics 1989;84:152-6.

18 Matteucci RM, Holbrook TL, Hoyt DB, et al. Trauma among Hispanic children: a population-based study in a regionalized system of trauma care. Am f Public Health 1995;85:1005-8.

19 Agran PF, Winn DG, Anderson CL, et al. Pediatric injury hospitalization in Hispanic children and non-Hispanic white children in southern California. Arch Pediatr Adolesc Med 1996;150:400-6.

20 Mobley C, Sugarman JR, Deam C, et al. Prevalence of risk factors for residential fire and burn injuries in the American Indian community. Public Health Rep 1994;109:702-5.

21 Grossman D, Sugarman J, Fox C, et al. Motor vehicle crash injury risk factors among American Indians. Accid Anal Prev 1997;29:313-19.

22 Campos-Outcalt D, Prybylski D, Watkins AJ, et al. Motor vehicle crash fatalities among American Indians and non-Indians in Arizona. Am $\mathcal{F}$ Public Health 1997;87:2825 .

23 Gallaher MM, Fleming DW, Berger LR, et al. Pedestrian and hypothermia deaths among Native Americans in New Mexico. Between bar and home. $\mathcal{F} A M A$ 1992;10:1345-8. 
24 Smith GS, Langlois JA, Buechner JS. Methodological issues in using hospital discharge data to determine the incidence of hospitalized injuries. Am f Epidemiol 1991; 134:1146-57.

25 Indian Health Service. Trends in Indian health. Rockville, MD: IHS, 1996.
26 Robertson LS. Community injury-control programs of the Indian Health Service: an early assessment. Public Health Indian Health Service:

27 Kunitz SJ. The history and politics of US health care policy for American Indians and Alaskan Natives. Am $\mathcal{F}$ Public Health 1996;86:1464-73.

Girl saved because "big brother" was watching her

Seven year old Kristian sprang into action when he realised his sister, Shanice, was choking on a peanut. He calmly dialled 999 for an ambulance and advice, which he relayed to his mother, Kerry. Kerry said that she panicked but Kristian kept her calm. In the past, Kristian had been told off for dialling 999 for fun (Daily Mail (London), December 1998).

\section{Update on an airbag fatality}

A man whose infant son was killed by an airbag in Ohio, USA, was fined $\$ 500$ and sentenced to two 12 hour days in jail for failing to turn off the device. The father will serve his time on the boy's birthday and on the anniversary of the crash, the municipal court judge determined. This is believed to be the first case of its kind in North America. The 29 year old father pleaded no contest on charges of vehicular homicide and running a red light in the death of his 2 month old son. They were in a pickup truck with a switch-off device for the passenger side airbag (and prominent warning signs) in the event a child was riding in that seat. The infant was in a rear-facing car seat (contributed by Amy Zierler).

\section{The child friendly Chrysler Neon?}

The version of the Chrysler Neon sold in the UK comes with twin front airbags so it is completely taboo to carry a rear-facing baby seat on the front passenger seat. Sadly, the seat belts in the back of the car are too short to accommodate the baby seats on the British market. It seems that Chrysler are correcting this. The current vehicle handbook advises that if you have trouble tightening the belt on the child restraint because the buckle or latch plate interferes with the belt path opening on the restraint (whatever all that means), disconnect the latch plate from the buckle and twist the short buckle-end belt several turns to shorten it.

One of the key ways of ensuring that child restraints are used correctly is to make them as user friendly as possible. Having to twist the belt several times every time you want to put the seat in the car does not seem to accord with this requirement! Roll on ISOFIX and its US equivalent. 